\title{
Natural killer cell-based adoptive immunotherapy eradicates and drives differentiation of chemoresistant bladder cancer stem-like cells
}

Margarida Ferreira-Teixeira ${ }^{1,2}$, Daniela Paiva-Oliveira ${ }^{1,2}$, Belmiro Parada ${ }^{1,3}$, Vera Alves ${ }^{4}$, Vitor Sousa $a^{5,6}$, Obinna Chijioke ${ }^{7}$, Christian Münz ${ }^{7}$, Flávio Reis ${ }^{1,2}$, Paulo Rodrigues-Santos ${ }^{4,8,9+}$ and Célia Gomes ${ }^{1,2,8,8^{*}+}$

\begin{abstract}
Background: High-grade non-muscle invasive bladder cancer (NMIBC) has a high risk of recurrence and progression to muscle-invasive forms, which seems to be largely related to the presence of tumorigenic stem-like cell populations that are refractory to conventional therapies. Here, we evaluated the therapeutic potential of Natural Killer (NK) cell-based adoptive immunotherapy against chemoresistant bladder cancer stem-like cells (CSCs) in a pre-clinical relevant model, using NK cells from healthy donors and NMIBC patients.

Methods: Cytokine-activated NK cells from healthy donors and from high-grade NMIBC patients were phenotypically characterized and assayed in vitro against stem-like and bulk differentiated bladder cancer cells. Stem-like cells were isolated from two bladder cancer cell lines using the sphere-forming assay. The in vivo therapeutic efficacy was evaluated in mice bearing a CSC-induced orthotopic bladder cancer. Animals were treated by intravesical instillation of interleukin-activated NK cells. Tumor response was evaluated longitudinally by non-invasive bioluminescence imaging.

Results: NK cells from healthy donors upon activation with IL-2 and IL-15 kills indiscriminately both stem-like and differentiated tumor cells via stress ligand recognition. In addition to cell killing, NK cells shifted CSCs towards a more differentiated phenotype, rendering them more susceptible to cisplatin, highlighting the benefits of a possible combined therapy. On the contrary, NK cells from NMIBC patients displayed a low density on NK cytotoxicity receptors, adhesion molecules and a more immature phenotype, losing their ability to kill and drive differentiation of CSCs. The local administration, via the transurethral route, of activated NK cells from healthy donors provides an efficient tumor infiltration and a subsequent robust tumoricidal activity against bladder cancer with high selective cytolytic activity against CSCs, leading to a dramatic reduction in tumor burden from $80 \%$ to complete remission.
\end{abstract}

Conclusion: Although pre-clinical, our results strongly suggest that an immunotherapeutic strategy using allogeneic activated NK cells from healthy donors is effective and should be exploited as a complementary therapeutic strategy in high-risk NMIBC patients to prevent tumor recurrence and progression.

Keywords: Bladder cancer, Cancer stem cells, Immunotherapy, Natural killer cells

\footnotetext{
* Correspondence: cgomes@fmed.uc.pt

${ }^{\dagger}$ Equal contributors

'Laboratory of Pharmacology and Experimental Therapeutics, Institute for

Biomedical Imaging and Life Sciences (IBILI), Faculty of Medicine, University

of Coimbra, Coimbra, Portugal

${ }^{2}$ CNC.IBILI, University of Coimbra, Coimbra, Portugal

Full list of author information is available at the end of the article
} 


\section{Background}

Bladder cancer (BC) is the most common malignancy of the urinary tract, and one of the leading causes of cancer death in Western countries [1,2]. Although the majority of the newly diagnosed cases are non-muscle-invasive tumors (NMIBC), up to $80 \%$ recur and a significant part progresses to therapy refractory muscle-invasive forms (MIBC) [3, 4].

A recent report from our group demonstrated that MIBC harbor distinct cell subsets reflecting molecular features of stem-like cells endowed with enhanced chemoresistance and tumor initiating ability [5]. In addition to the inefficacy of conventional chemotherapy towards bladder cancer stem-like cells (CSCs), we also showed that a short-term exposure to cisplatin induced a phenotypic cell state transition to an adaptive stem-like phenotype, providing evidence for the tumor plasticity and spontaneous switching between cell states when subjected to stressful conditions such as chemotherapy [5]. Evidence from other groups supports our findings, reinforcing the hypothesis of a driver role of those cells in the frequent relapses of $\mathrm{BC}$, as well as a fuel to the progression towards invasive forms $[6,7]$. Therefore, the development of therapeutic strategies aimed to target cancer stemness is essential to prevent tumor relapse and progression, and represents an important challenge in $\mathrm{BC}$ management.

Natural Killer (NK) cells are important players of the innate immune system with a strong cytolytic activity against virus-infected or neoplastic cells [8] without prior immune sensitization, which make them appealing therapeutic effectors against cancer [9]. These cells secrete inflammatory cytokines and chemokines that subsequently shape the innate and adaptive immune response by promoting differentiation, activation and recruitment of accessory immune cells to the tumor site $[10,11]$. The biological activity of NK cells is regulated by the dynamic balance between activating and inhibitory signals provided by the interaction with the target cells, and by soluble factors released in the tumor microenvironment, which together dictate their efficacy [12]. NK cells express a variety of activating receptors, including the NK group 2 member D (NKG2D), the DNAX accessory molecule-1 (DNAM-1), and the natural cytotoxicity receptors (NCRs: NKp30, NKp44, and NKp46), that provide activating signals upon binding to stressinduced ligands that are expressed in tumor, but not in normal cells. The inhibition of NK cells is mediated by the inhibitory killer-cell immunoglobulin-like receptors or NKG2A/CD94 that recognize classical or nonclassical HLA class I molecules, respectively, which are often lost or reduced in malignant cells [13, 14]. Contrarily to conventional chemotherapy, NK cells appear to recognize and kill undifferentiated stem-like cells $[15,16]$ by virtue of their ability to target non-dividing cells and due to the low expression of MHC class I molecules and possible up-regulation of stress-induced activation ligands [17-19].

These findings, along with the relevance of CSCs in $\mathrm{BC}$ progression and the inefficacy of current therapies, prompted us to evaluate the therapeutic potential of adoptive NK cell-based immunotherapy in the eradication of competent CSCs and its impact on tumor progression, an approach that is yet to be explored in BC.

\section{Methods \\ Cell lines}

Human BC (HT-1376 and UM-UC3) and the leukemic (K562) cell lines (American Type Culture Collection, Manassas, VA, USA) were cultured in RPMI 1640 medium (Gibco, Scotland, UK) supplemented with $10 \%$ heat inactivated fetal bovine serum (FBS), $200 \mathrm{mM}$ of Lglutamine (Sigma, St. Louis, USA), and penicillin (100 IU/mL)-streptomycin $(100 \mathrm{mg} / \mathrm{mL})$ (Gibco, Scotland, UK), at $37{ }^{\circ} \mathrm{C}$ in a $5 \% \mathrm{CO}_{2}$ incubator. CSCs were isolated from the $\mathrm{BC}$ cell lines as described previously [5].

\section{Isolation of NK cells from healthy donors and bladder cancer patients}

Polyclonal NK cells were isolated from healthy donor (HD, $\mathrm{n}=30$, mean age: 45 years old) buffy coats provided by the Portuguese Blood and Transplantation Institute or from the blood of $\mathrm{BC}$ patients after receiving informed consent and approval by the Institutional Review Board of Coimbra University Hospital (Approved ID: 018-CE-2016). BC patients' blood was collected from a cohort of 10 male patients (mean age of 70 years) classified as Ta high-grade NMIBC before surgical treatment. Peripheral blood mononuclear cells were separated by density gradient centrifugation on FicollHypaque (GE Healthcare, Uppsala, Sweden). NK cells were subsequently isolated by negative selection using the NK-cell isolation kit (Miltenyi Biotec) according to the manufacturer's instructions. Purified NK cells were cultured in complete RPMI-1640 medium $\left(10^{6} / \mathrm{mL}\right)$ supplemented with $10 \%$ heat inactivated FBS, $200 \mathrm{mM}$ of L-glutamine (Sigma), penicillin (100 IU/mL), and streptomycin $(100 \mathrm{mg} / \mathrm{mL})$. For activation and expansion, NK cells were incubated with the interleukins IL-2 (250 IU/mL) and IL-15 (0.1 mg/mL) (Peprotech, Rocky Hill, NJ, USA) for 24 and $48 \mathrm{~h}$. The purity of the isolated $\mathrm{CD}^{-} \mathrm{CD}^{-} 6^{+} \mathrm{NK}$ cell populations was $>95 \%$ in all experiments.

\section{Immunophenotyping of NK cells isolated from healthy donors and bladder cancer patients}

NK cells were stained with fluorochrome-conjugated monoclonal antibodies against the following human surface antigens: CD56-PE-Cy7, CD16-APC-H7, CD3/ 
CD14/CD19-PerCP-CY5.5, CD94/CD27/CD62L-FITC, NKG2C/NKp30/NKp46/NKG2D-APC, CD11b-PB, and NKG2A/NKp44/NKp80-PE (all purchased from Biolegend, San Diego, CA, USA). For intracellular staining, cells were washed, fixed, and permeabilized with Fix \& Perm cell fixation and permeabilization kit (Invitrogen, Carlsbad, CA, USA) and stained with IL-4/TGF- $\beta$-FITC, TNF- $\alpha-P E, I L-10-A P C$, and IFN- $\gamma$-PB. Appropriate isotype controls were used. A minimum of 100,000 events were acquired using a FACSCanto II flow cytometer (BD Biosciences, San Jose, CA, USA) and analyzed with the FlowJo analysis software (Tree Star, Inc., Ashland, USA). Results were expressed as the percentage of positively stained cells in the NK cell gate.

\section{Immunophenotyping of BC cells}

Single-cell suspensions of parental and corresponding sphere-forming cells were stained for $30 \mathrm{~min}$ at $4{ }^{\circ} \mathrm{C}$ with fluorescent conjugated monoclonal antibodies against HLA-ABC (clone w6/32, BioLegend), MICA/B (clone 6D4, BioLegend), ULBP1 (clone 170818, R\&D Systems, Minneapolis, MN, USA), CD48 (clone 394607, R\&D Systems), Nectin-2/CD112 (clone 610603, R\&D Systems), CD155/PVR (clone 300907, R\&D Systems), and Fas/CD95 (clone 2R2, eBiosciences, San Jose, CA, USA). For experiments with the supernatant of NK cells (NK-SN), spheres were previously incubated for $4 \mathrm{~h}$ with the supernatants of IL-2- and IL-15-activated NK cells before phenotyping. Appropriate isotype-matched controls were run with each experiment. Samples were analyzed using a FACSCanto II cytometer. A minimum of 100,000 events were collected and analyzed using the FlowJo software.

\section{CD107a degranulation and cytokine production}

Freshly and IL-2/IL-15-activated NK cells $\left(10^{6}\right.$ cells) collected from HDs were co-cultured with target cells at an effector-to-target (E:T) ratio of 3:1 in U-bottomed 96-well plates for $4 \mathrm{~h}$ in a $5 \% \mathrm{CO}_{2}$ incubator with PE-conjugated anti-CD107a (H4A3, BioLegend) and Brefeldin A (Golgistop, BD). Stimulus with $25 \mathrm{ng} / \mathrm{mL}$ PMA plus $250 \mathrm{ng} / \mathrm{mL}$ ionomycin was used as a positive control and NK cells alone were used as a negative control. Cultured cells were then stained with fluorochromeconjugated monoclonal antibodies against human blood surface antigens: CD3 PerCP/Cy5.5 (clone HIT3a), CD14 PerCP/Cy5.5 (clone M5E2), CD19 PerCP/Cy5.5 (clone HIB19), CD16 FITC (clone 3G8), and CD56 APC (clone HCD56), all purchased from BioLegend. The percentage of $\mathrm{CD}^{-} \mathrm{CD} 56^{+} \mathrm{NK}$ cells positive for CD107a was calculated. All analyses were performed in duplicate using BD FACSCanto II and FlowJo analysis software.
Cytokines produced by $48 \mathrm{~h}$ IL-2/IL-15-activated NK cells co-cultured with tumor cells at an E:T ratio of 10:1 were measured using ELISA kits according to the manufacturer's instructions (granzyme B and IFN- $\gamma$ : Abcam, Cambridge, UK; and TNF- $\alpha$ : R\&D Systems, MN, USA).

\section{Chromium-51 $\left({ }^{51} \mathrm{Cr}\right)$-release assay}

Target cells were loaded for $1 \mathrm{~h}$ with $50 \mu \mathrm{Ci}$ of ${ }^{51} \mathrm{Cr}$ (PerkinElmer, Massachusetts), washed twice and incubated with fresh or activated NK cells at different E:T ratios (1:1, 3:1 and 10:1) in $200 \mu \mathrm{L}$ of complete RPMI in 96-well Ubottom tissue culture plates at $37^{\circ} \mathrm{C}$ in a $5 \% \mathrm{CO}_{2}$.

After a 4-h incubation period, the supernatants were harvested and counted for released radioactivity in a gamma counter (CRC-55tW Capintec), within a ${ }^{51} \mathrm{Cr}$ sensitivity energy window $(300-400 \mathrm{keV})$. The specific lysis of target cells was calculated as follows: Percentage of specific lysis $=($ experimental release - spontaneous release) $/$ (maximum release - spontaneous release) $\times 100$. Spontaneous release was calculated from target cells without effector cells. Maximum release was determined by incubating target cells with $4 \%$ SDS detergent. In all experiments, the spontaneous release was $<20 \%$ of maximum release.

For NK cells blocking receptor experiments, activated NK cells were pre-incubated with $10 \mu \mathrm{g} / \mathrm{mL}$ of antiNKG2D (clone 149810, R\&D Systems), $10 \mu \mathrm{g} / \mathrm{mL}$ of anti-DNAM-1 (clone 102511, R\&D Systems), and $0.5 \mu \mathrm{g} / \mathrm{mL}$ of anti-FasL (clone ZB4, Merck Millipore, Germany), individually or in combination, before coculture with tumor target cells.

\section{NK cell supernatant assays}

Both parental and CSCs were cultured for $4 \mathrm{~h}$ with the supernatant harvested from 48-h IL-2/IL-15-activated NK cells from HDs or BC patients. Thereafter, tumor cells were assayed for aldehyde dehydrogenase (ALDH) activity, expression of stemness-related markers and cell surface ligands for NK receptors and chemosensitivity to cisplatin.

\section{Aldefluor assay}

The activity of ALDH in tumor cells was measured using the Aldefluor kit (Stem Cell Technologies, Vancouver, $\mathrm{BC}$, USA), according to the manufacturer's instructions. FACS was performed on a BD FACSCanto II flow cytometer. Data was analyzed with the FlowJo software.

\section{Gene expression by real-time quantitative PCR analysis (RT-qPCR)}

Total RNA from sphere-forming and parental cells was extracted using the ReliaPrep RNA Cell Miniprep System (Promega) following the manufacturer's instructions. The quantity and quality of isolated RNA was measured by the 
ND-1000 spectrophotometer (NanoDrop Technologies). Reverse transcription from $1 \mu \mathrm{g}$ of total RNA was performed using NZY First-Strand cDNA Synthesis kit (Nzytech) and subsequent RT-qPCR for SOX2, ABCG2, ABCB1, ALDH1A1, ALDH2, CD44, CD47, and KRT14 was performed as previously described [5]. Primers used on RT-qPCR reaction are listed in Additional file 1: Table S1. mRNA expression was normalized to three housekeeping genes: 18S, GAPDH, and HRPT-1 using the $\triangle \triangle \mathrm{Ct}$ method and Bio-Rad CFX Manager ${ }^{\mathrm{TM}} 3.0$ software.

\section{Chemosensitivity to cisplatin}

Cells were treated with increasing concentrations of cisplatin (Teva Pharma, Portugal) ranging from 1 to $100 \mu \mathrm{M}$ over $48 \mathrm{~h}$. Cell viability was analyzed using the standard MTT [3-(4,5-dimethylthiazol-2-yl)-2,5-diphenyltetrazolium bromide] (Sigma) assay as previously described [5]. Cell viability was expressed as the percentage of absorbance values of the treated cells related to the untreated control wells considered as $100 \%$.

\section{Bladder tumor specimens and immunohistochemistry} Bladder tumor samples were obtained from 25 patients (19 males and 6 females) by transurethral resection at Coimbra University Hospital, following appropriate informed consent and ethical regulatory approval (Approved ID: 018-CE-2016). Tumors at initial diagnosis were stratified into non-muscle-invasive low $(\mathrm{n}=15)$ and high $(n=7)$ grade and muscle-invasive tumors $(n=3)$ by a pathologist, according to the 2004 WHO criteria [20]. Formalin-fixed paraffin-embedded tissue blocks were sectioned at 3- $\mu \mathrm{m}$ thickness and incubated in a BenchMark Ultra Ventana, with a primary antibody against CD56, a surface marker for NK cells, clone 123C3 (1:50, Roche), for $30 \mathrm{~min}$ at $37{ }^{\circ} \mathrm{C}$, and reaction signal was developed with 3-3'-diaminobenzidine tetrahydrochloride chromogen. Standard procedures were used for visualization and the intensity and percentage of positive staining was registered. Two investigators blinded to the data reviewed all slides independently.

\section{Animal studies}

Animal studies were approved by the Organization Responsible for Animal Welfare of the Faculty of Medicine of Coimbra (Approved ID: ORBEA/91/2015/08) and were performed according to National and International guidelines on animal experimentation. Female nude mice (Swiss nu/nu), 6-8 weeks old (Charles River Laboratories, Barcelona, Spain) were housed under pathogen-free conditions in individual ventilated cages. The subcutaneous tumor model was induced by subcutaneous injection into the lower flank of $1 \times 10^{6}$ of $\mathrm{Luc}^{+}$ HT-1376 cells suspended in $100 \mu \mathrm{L}$ of a 1:1 PBS/Matrigel mixture. The orthotopic model that more closely resembles the clinical and histopathological features of primary MIBC was developed by intravesical instillation of Luc $^{+}$HT-1376 cells as previously described [5]. Bioluminescent images were taken $24 \mathrm{~h}$ post-implantation and every 3 days to monitor engraftment and growth of tumor cells using an IVIS Lumina XR (Caliper LifeSciences, Hopkinton, MA, USA) after intraperitoneal injection with D-luciferin (150 mg/kg, Synchem, BHg, Germany) with the animals under anesthesia $(100 \mathrm{mg} / \mathrm{kg}$ ketamine and $2.5 \%$ of chlorpromazine solution). Quantification of bioluminescent signals was performed using the living image software version 4.10 (Xenogen). Values are expressed as photons $/ \mathrm{sec} / \mathrm{cm}^{2} / \mathrm{sr}$. Subcutaneous tumors started the treatment on day 6 post-implantation by intratumoral inoculation of NK cells activated for $48 \mathrm{~h}(5 \times$ $\left.10^{6} / 50 \mu \mathrm{L}\right)$ from HDs twice a week over 2 weeks.

Animals bearing subcutaneous or orthotopic tumors were treated twice a week with healthy 48-h activatedNK cells $\left(5 \times 10^{6} /\right.$ mouse $)$ via intratumoral and intravesical instillation, respectively, over 2 weeks. NK cells were washed prior to administration and resuspended in PBS. Tumor progression was monitored by bioluminescent images 3 days after each treatment. Animals were sacrificed after treatments or when presenting hematuria or lost $20 \%$ of initial body weight. Residual tumors were excised and processed into paraffin blocks for immunohistochemistry analysis of CD56 clone 123C3 (1:50, Roche) and for two CSC-related markers, SOX-2 (clone D6D9, 1:100, Cell Signaling) and ALDH2 (clone EPR4493, 1:100, Abcam) as described above for clinical samples.

\section{Statistical analysis}

Data are reported as the means \pm SEM of the indicated number of experiments. Statistical analysis and graphic illustrations were performed using GraphPad Prism 6.0 software (San Diego, CA). Paired two-tailed Student's t-tests, ANOVA, and Tukey's tests were used to calculate $P$ values. A $P$ value of less than 0.05 was considered significant.

\section{Results}

Activated-NK cells from healthy donors are highly effective against chemoresistant bladder cancer stem-like cells

The functional activity of NK cells from HDs against parental cells and CSCs was evaluated by measuring CD107a degranulation, release of cytokines, and lysis of target cells following a $4 \mathrm{~h}$ co-culture period. Freshly isolated NK cells were in the resting state in all E:T ratios tested, as indicated by the low CD107a degranulation rates, displaying weakly cytolytic activity against any cell line including the MHC class I-negative K562 cells. Upon stimulation with IL-2/IL-15, NK cells enhanced their functionality and cytotoxicity against either parental 
cells or CSCs, as demonstrated by the enhanced CD107a degranulation rates, and release of IFN- $\gamma$, TNF- $\alpha$, and granzyme B (a lytic granule) as compared to fresh NK cells (Fig. 1a, b). During the 24 and $48 \mathrm{~h}$ of activation, the percentage of viable NK cells decreased to $20-30 \%$.

The cytolytic activity of NK cells, measured by the ${ }^{51} \mathrm{Cr}$-release assay, increased with increasing E:T ratio and reached a specific lysis greater than $70 \%$ for an E:T ratio of 10:1 in both cell subsets upon $48 \mathrm{~h}$ activation with IL-2/IL-15 (Fig. 1c). No significant differences were obtained between CSCs and parental cells, indicating equal susceptibility of $\mathrm{BC}$ cells to activated NK cells lysis.

Flow cytometry analysis of the various receptors involved in NK cell effector functions showed a significant up-regulation of the NCRs NKp44 $(2.00 \pm 1.16 \%$ vs. $26.33 \pm 3.84 \%, P<0.01)$ and NKp30 $(0.12 \pm 0.02 \%$ vs. $2.68 \pm 0.33 \%, P<0.01)$, and of the NKG2D $(65.00 \pm$

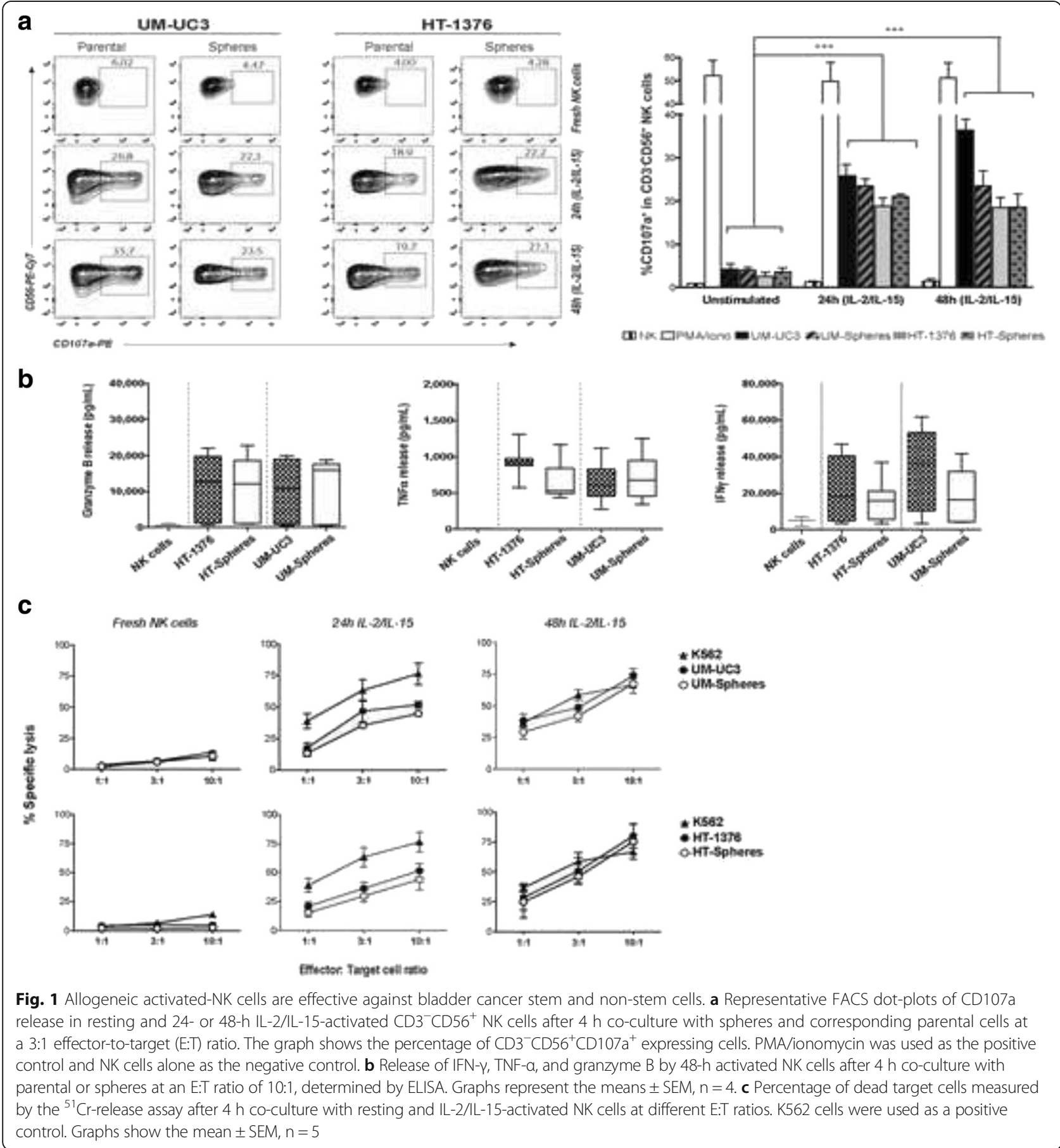


$9.45 \%$ vs. $96.33 \pm 1.76 \%)$ and DNAM-1 (78.67 $\pm 3.66 \%$ vs. $92.25 \pm 1.65 \%$ ) activating receptors upon $48 \mathrm{~h}$ activation, relative to resting NK cells (Additional file 2: Figure S1), indicating the crucial role of stimulatory cytokines in NK cell antitumor properties.

Moreover, the distribution of gated $\mathrm{CD}^{+} 6^{+} \mathrm{CD}^{-} \mathrm{NK}$ cells with regard to CD16 expression changed upon stimulation with IL-2/IL-15, resulting in a significant increase in the $\mathrm{CD} 16^{-}$subpopulation in relation to resting NK cells. The median percentage of CD $56^{\text {bright }} \mathrm{CD} 16^{-}$, which in freshly NK cells was of $2.68 \pm 0.20 \%$ (2.34-3.29\%), increased to $4.32 \pm 0.21 \%$ $(3.98-4.85 \%)$ and to $8.57 \pm 1.02 \%(6.64-10.10 \%)$ upon $24-$ and 48 -h cytokine activation, respectively.
No significant changes were observed in the percentage of $\mathrm{CD} 56^{\mathrm{dim}} \mathrm{CD} 16^{+}$cells.

\section{Bladder CSCs display increased expression of ligands recognized by NK cell activating receptors}

To evaluate the ability of $\mathrm{BC}$ cells to stimulate NKmediated cytotoxicity, both parental cells and CSCs were characterized regarding the expression of ligands that engage activating and inhibitory NK receptors. Both parental cells and CSCs expressed activating ligands involved in NK recognition, namely MICA/B and ULBP-1 ligands for NKG2D-activating receptor and PVR and Nectin-2 for DNAM-1, as well as the Fas death receptor (Fig. 2a). Interestingly, all activating ligands were found

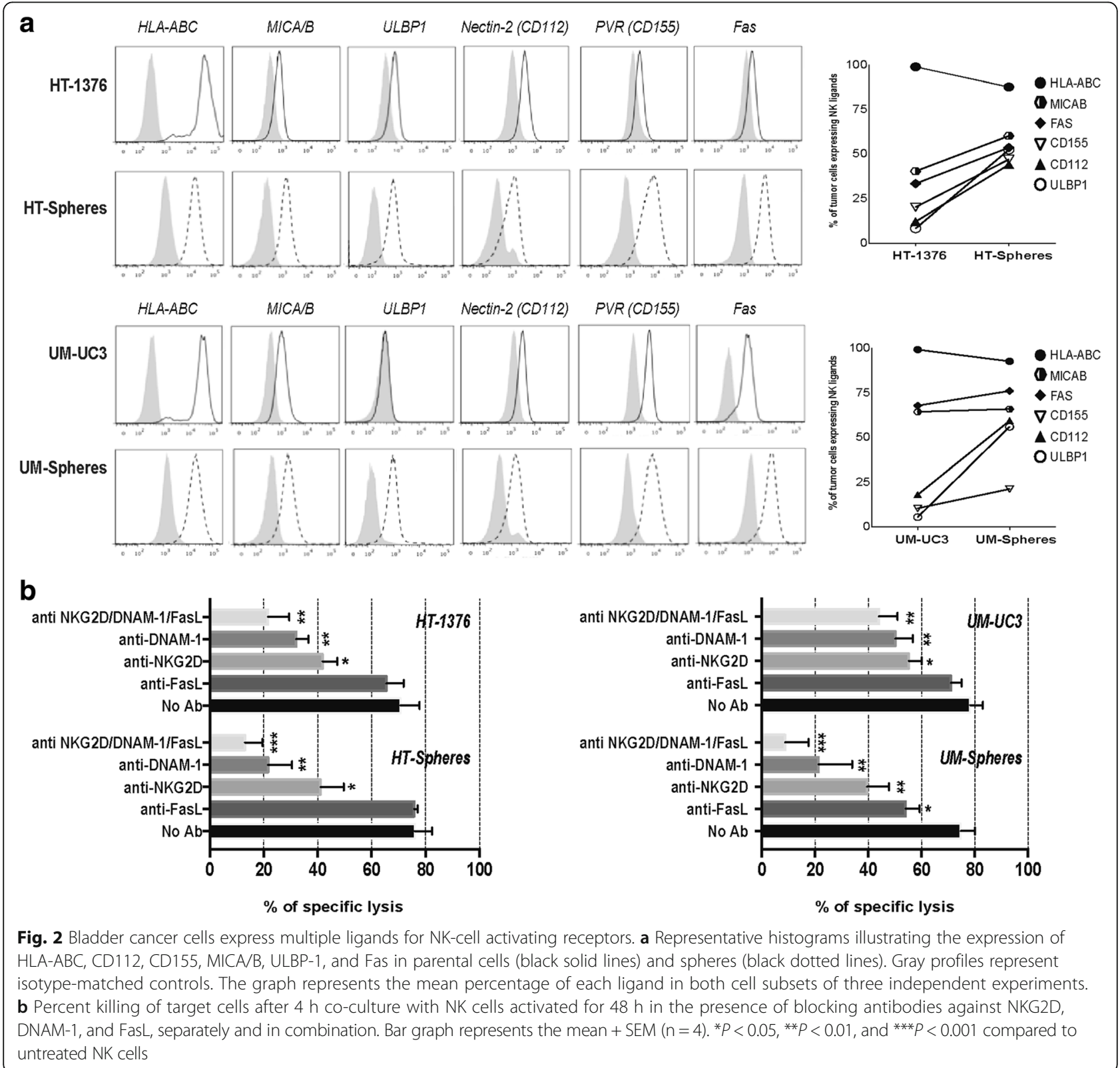


more highly expressed in the CSC subsets in comparison to corresponding parental cells. The HLA-class I molecules (HLA-ABC), which play a major role in NK cell inhibition, were expressed in both $\mathrm{BC}$ cell lines and slightly decreased in spheres.

\section{NKG2D and DNAM-1 activating receptors mediate bladder CSC lysis}

To identify the contribution of the different activating receptors behind NK cell recognition of target cells, we performed blocking studies using specific monoclonal antibodies. As indicated by the killing assay (Fig. 2b), blocking NKG2D $(P<0.05)$ and DNAM-1 $(P<0.01)$ receptors impaired the overall cytolytic activity of NK cells against both BC cell subsets. Additionally, Fas-L blocking decreased the ability of NK cells to kill the stem-like fraction of the UM-UC3 cell line in agreement with the high surface expression levels of Fas in these cells. The combined mAb-mediated blocking of NKG2D, DNAM1 , and Fas-L receptors almost completely abrogated NK cell-mediated killing of spheres from the two BC cell lines, in agreement with the higher density of ligands interacting with these specific NK-activating receptors.

\section{Supernatants from NK cells induce differentiation and sensitize CSCs to cisplatin}

In addition to increased chemoresistance, CSCs are characterized by their ability to self-renew and differentiate. We tested whether NK cells could induce CSCs towards a more differentiated phenotype rendering them susceptible to chemotherapy.

Therefore, spheres were incubated with the supernatants of activated-NK cells for $4 \mathrm{~h}$, followed by analysis of stemness related markers previously identified [5]. The ALDH activity, considered a functional readout of stemness, decreased by $60 \%$ in spheres after $4 \mathrm{~h}$ incubation with NK supernatants (Fig. 3a). Accordingly, the transcript levels of two ALDH isoforms responsible for ALDH activity (ALDH1A1 and ALDH2) were also down regulated in both CSC populations (Fig. 3b). The mRNA expression levels of other stem cell-related markers, including pluripotency factors (SOX2, POU5F1, and NANOG), urothelial basal cell-specific markers (CD44, CD47, and KRT14), and drug resistance-related transporters (ABCG2 and $A B C B 1$ ), were also significantly downregulated in HT-1376 spheres. A similar trend, although not significant, was noticed in UM-UC3 spheres. No significant transcription changes were observed in corresponding parental cells (data not shown). Additionally, pre-treatment with the NK cell supernatant sensitized CSCs towards cisplatin, a drug currently used in the treatment of MIBC, as compared to non-pretreated cells (Fig. 3c).

\section{NK cells from bladder cancer patients display low} expression of NCRs and fail to mediate CSCs lysis Next, we analyzed the phenotypic status and functionality of NK cells collected from the peripheral blood of high-grade NMIBC patients with high risk of recurrence. NK cells displayed decreased responsiveness to cytokine stimulation, as indicated by the overall lower specific lysis observed in both cell subsets comparatively to activated-NK cells from HDs (Fig. 4a, b), with considerably reduced cytotoxicity against spheres $(P<0.01)$, contrary to healthy NK cells, which displayed an equal capacity to kill stem and parental cells (Table 1). Phenotypic analysis showed a reduced expression of NKp30, NKp44, and the co-receptor NKp80 in patient NK cells, as compared with HDs (Fig. 4c). The expression of the adhesion molecule CD62L and the terminal differentiation marker CD57 was significantly decreased in NK cells from BC patients. Furthermore, NK cells from patients showed up-regulation of the immunosuppressive anti-inflammatory cytokines TGF- $\beta$, IL- 4 , and IL-10, and down regulation of pro-inflammatory cytokines TNF- $\alpha$ and IFN- $\gamma$, in agreement with the impaired NK cell activity (Table 1).

Moreover, exposure of CSCs to NK supernatants derived from $\mathrm{BC}$ patient cells did not decrease the expression of stemness-related markers in spheres. Rather, a trend was observed towards up-regulation of the majority of analyzed genes, suggesting that NK cells release factors that maintain or exacerbate the stemness features of tumor cells (Fig. 4d).

To further evaluate whether tumor-infiltrating NK cells might indeed represent an ongoing anti-immunity response in $\mathrm{BC}$, we analyzed the expression of $\mathrm{CD} 56^{+}$ NK cells in a panel of human BC samples classified as low- and high-grade NMIBC and MIBC at diagnosis. Our results revealed a small percentage of infiltrating $\mathrm{CD} 6^{+} \mathrm{NK}$ cells within tumors in all tumor stages, indicating these tumors are not infiltrated by NK cells, being unlikely to greatly contribute to the elimination of tumor cells (Fig. 4e).

\footnotetext{
Adoptive transfer of healthy activated-NK cells display anti-tumor activity in bladder cancer xenografted models Given the considerable low cytotoxic activity of NK cells from BC patients, we focused on the anti-tumoral activity of NK cells from HD in animal models induced by xenotransplantation of HT-1376 cells. The HT-1376 cell line contains a subpopulation of CSCs, as previously demonstrated by the presence of an $\mathrm{ALDH}^{+}$population with sphere-forming ability, and forms an orthotopic heterogeneous tumor resembling the clinical condition of MIBC comprising stem-like and proliferative differentiated cell populations, as previously demonstrated by our group [5].
} 
a
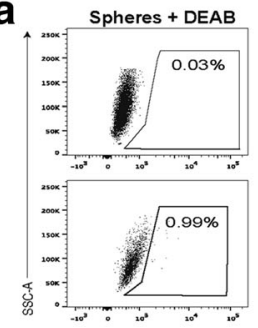$$
\text { FIT }
$$
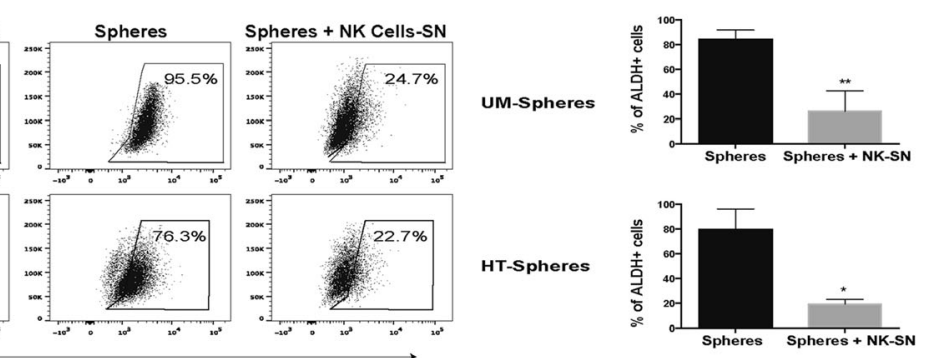

b
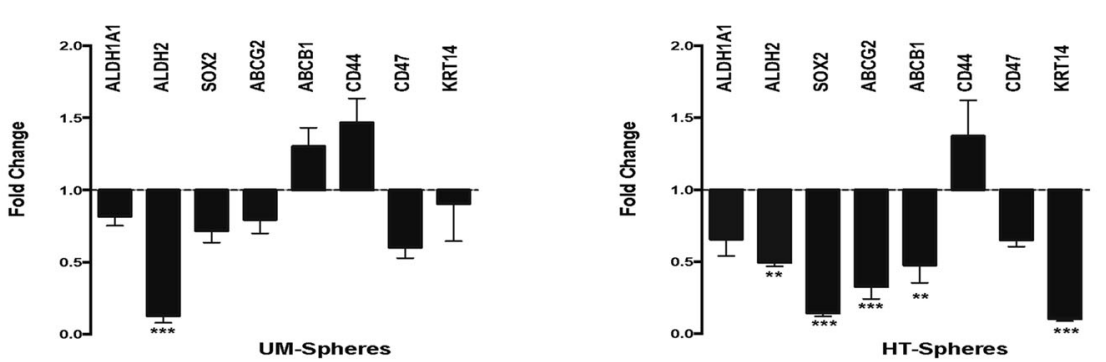

C
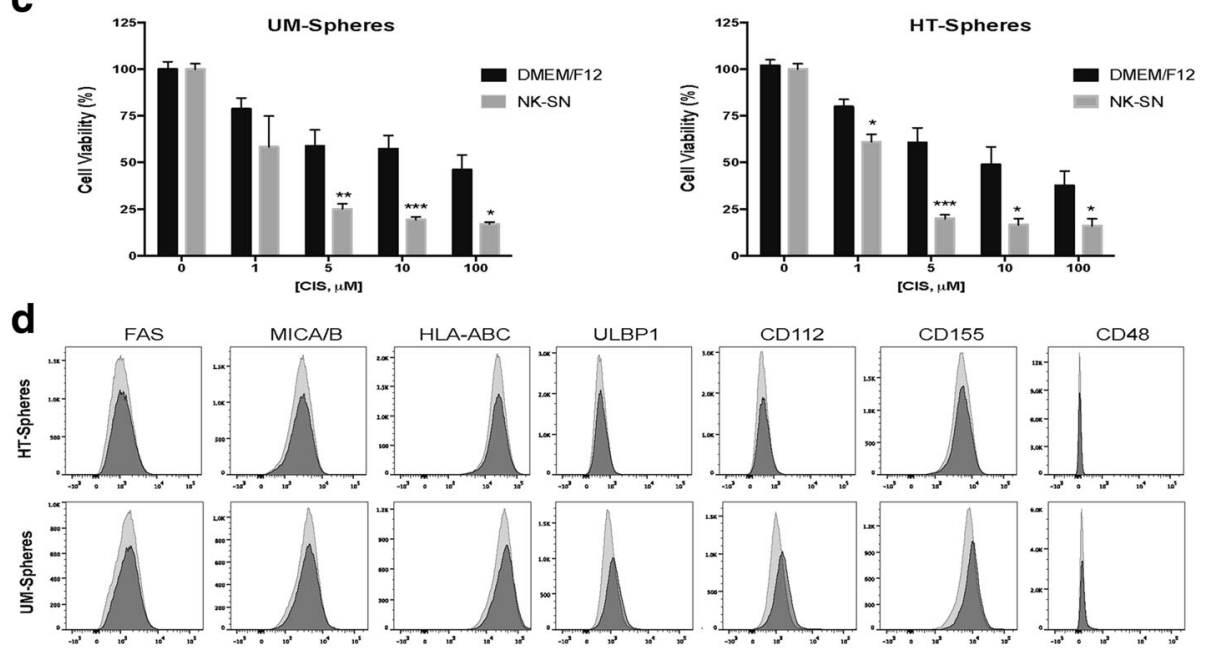

DMEM/F12

NK Cells-SN

Fig. 3 NK cell-derived supernatant (SN) promotes the differentiation of cancer stem-like cells and increases their sensitivity to cisplatin. a Representative flow cytometry analysis of ALDH enzymatic activity in spheres after $4 \mathrm{~h}$ with NK-derived SN. The ALDH inhibitor DEAB was used as a negative control to establish the baseline fluorescence of the cells. Bar graph shows the percentage of $\mathrm{ALDH}^{+}$cells (mean $\pm \mathrm{SEM}, \mathrm{n}=3$ ). $\mathbf{b}$ Fold-change $\mathrm{mRNA}$ expression levels of stemness-related markers in spheres treated with NK-SN relative to untreated spheres set as 1 (mean \pm SEM, $\mathrm{n}=5$ ). c Cytotoxic effects of cisplatin in spheres cultured in DMEM/F12 (black bars) and upon culture with NK-SN (gray bars) for $4 \mathrm{~h}$ determined by the MTT assay. The percentage of viable cells was normalized respective to untreated cells (mean $\pm S E M, n=3$ ). $\mathbf{d}$ Representative histograms illustrating the expression of specific ligands on the surface of spheres cultured in DMEM/F12 medium (gray histograms) or previously incubated with NK-SN for $4 \mathrm{~h}$ (black histograms). ${ }^{*} P<0.05$, ${ }^{* *} P<0.01$, and ${ }^{* *} P<0.001$ represents spheres in NK-SN vs. spheres in regular DMEM/F12

First, we evaluated the antitumor activity of NK cells in mice bearing localized subcutaneous tumors. The treatment started 6 days after cell inoculation and was performed twice a week by intratumoral injection of $5 \times 10^{6}$ activated-NK cells. An immediate and progressive decrease in the tumor size was observed, being totally abolished after the fourth administration (Fig. 5a). At that time, the treatment was finished and the animals were monitored for up to 2 weeks, and no tumor relapse was observed. Thereafter, we tested the same approach, but in an organ-specific microenvironment using an orthotopic model (Fig. 5b). NK cells were instilled intravesically into the bladder lumen 4 weeks after tumor cells implantation. The treatment resulted in a progressive decrease of tumor burden by $80 \%$ after the fourth inoculation with total remission in one of the five animals treated.

The immunostaining of residual tumors showed a high degree of $\mathrm{CD} 6^{+}$tumor-infiltrating NK cells, and a marked reduction of two stemness markers (SOX-2/ ALDH2) expression in treated tumors, compared to 
a
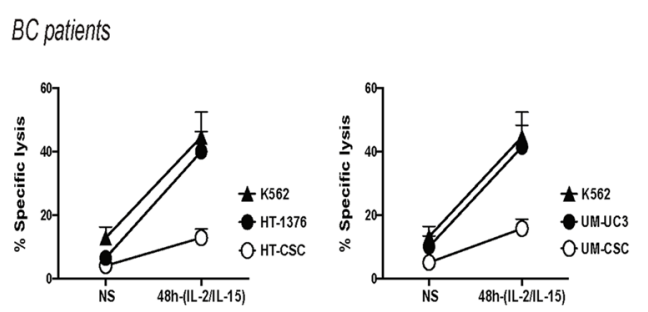
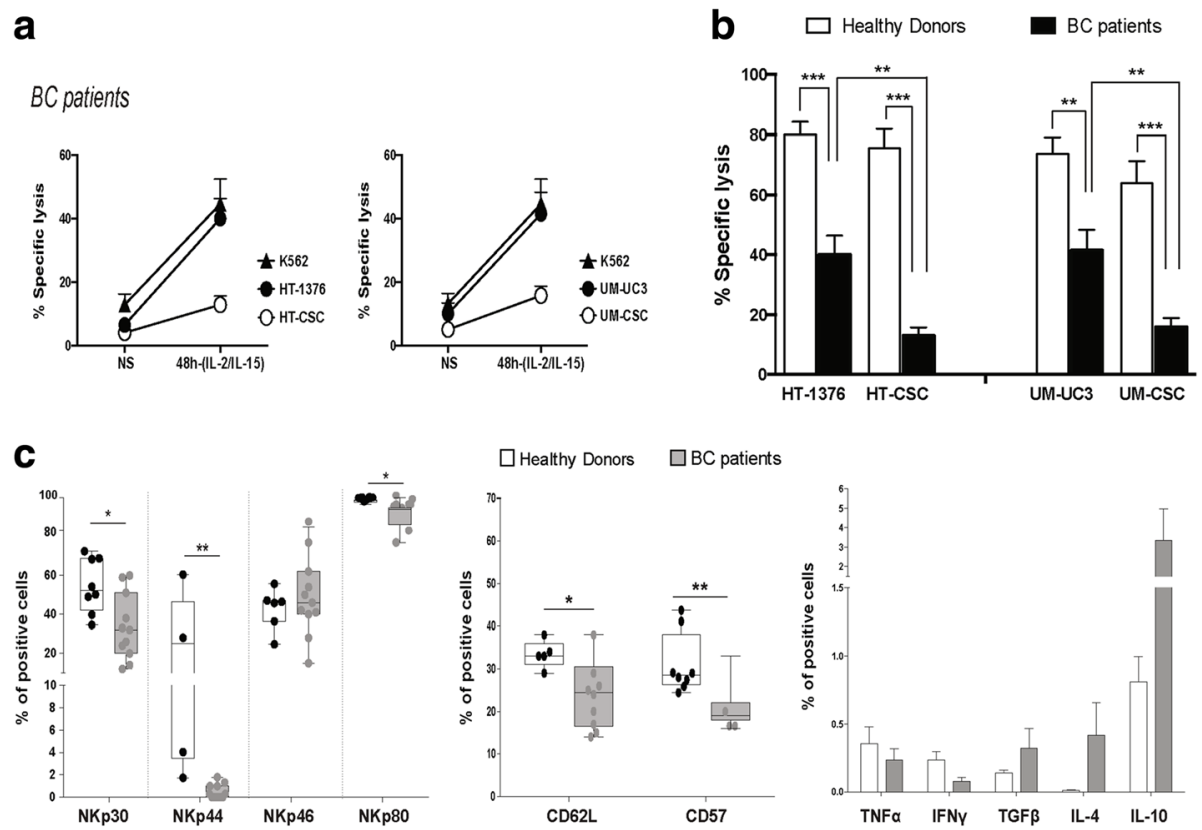

d
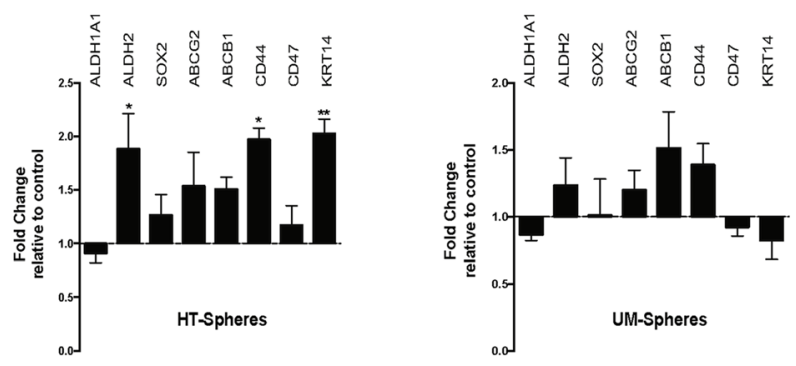

e

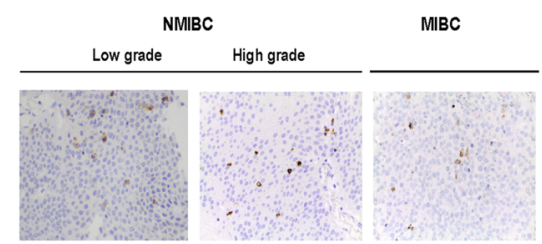

Fig. 4 NK cells from bladder cancer (BC) patients have impaired cytolytic activity and are ineffective against cancer stem-like cells. a Cytolytic activity of resting and IL-2/LL-15-activated NK cells after $48 \mathrm{~h}$ of activation derived from BC patients against BC parental cells and spheres after $4 \mathrm{~h}$ co-culture at a 10:1 ratio measured by the ${ }^{51} \mathrm{Cr}$ release assay. $\mathbf{b}$ Comparative analysis of lytic activity of NK cells derived from HDs and BC patients against spheres and parental cells. c Flow cytometry analysis of the natural cytotoxicity receptors, adhesion molecule CD62L, differentiation marker CD57, and the cytokine profile of BC patient (gray boxes, $n=10$ ) and $\mathrm{HD}$ (white boxes, $n=8$ ) NK cells. Graphs represent the percentage of positive cells (mean \pm SEM). ${ }^{*} P<0.05,{ }^{* *} P<0.01$, and ${ }^{* * *} P<0.001 \mathrm{HD}$ versus $\mathrm{BC}$ patients. $\mathbf{d}$ Fold-change mRNA expression levels of stemness-related markers in spheres treated with NK-SN from BC patients relative to untreated spheres set as 1 (mean $\pm S E M, n=5$, ${ }^{*}<0.05$ and ${ }^{* *} P<0.01$ ). e Immunohistochemical staining for CD56 in serial sections of tumoral tissue from BC patients with non-muscle-invasive, low grade; non-muscle-invasive, high grade; and muscle-invasive tumors. Original magnification: $\times 400$

untreated controls (Fig. 5c), confirming the CSCtargeting ability of locally administered NK cells in an organ-specific microenvironment.

\section{Discussion}

A major challenge in $\mathrm{BC}$ treatment is the risk of progression to muscle-invasive forms or metastatic disease, a process that appears to be strongly related to the presence of CSCs that are resilient to current conventional therapies. We provided evidence that both stem and non-stem cells can be recognized and effectively killed by ex vivo-activated allogeneic NK cells from HDs, but not from BC patients.

Compared with resting cells, cytokine-activated NK cells displayed an increased density of major activating receptors, crucial for NK cell cytokine production and 
Table 1 Profile of NK cells derived from healthy donors and bladder cancer patients

\begin{tabular}{lll}
\hline & Healthy donors & Bladder cancer patients \\
\hline${ }^{51}$ Cr-release assay & & \\
HT-1376 & $80.12 \pm 4.14 \%$ & $38.67 \pm 8.88 \% \%^{* * *}$ \\
HT-1376 spheres & $75.44 \pm 6.53 \%$ & $11.74 \pm 3.12 \% \%^{* * *}$ \\
UM-UC3 & $73.69 \pm 5.40 \%$ & $41.38 \pm 10.71 \%^{* *}$ \\
UM-UC3 spheres & $67.52 \pm 7.61 \%$ & $18.19 \pm 4.17 \% \%^{* * *}$ \\
NCRs & & \\
NKp30 & $54.68 \pm 4.98 \%$ & $33.55 \pm 5.09 \%^{*}$ \\
NKp44 & $26.35 \pm 9.18 \%$ & $0.52 \pm 0.20 \% \%^{* * *}$ \\
NKp46 & $42.93 \pm 3.70 \%$ & $49.09 \pm 6.09 \%$ \\
NKp80 & $98.34 \pm 0.32 \%$ & $91.36 \pm 2.20 \% \%^{* *}$ \\
CD62L & $33.40 \pm 1.44 \%$ & $24.30 \pm 2.57 \% \%^{*}$ \\
CD57 & $31.08 \pm 2.54 \%$ & $20.86 \pm 2.14 \% \%^{* *}$ \\
Cytokines & & \\
TNF-a & $0.36 \pm 0.13 \%$ & $0.23 \pm 0.08 \%$ \\
IFN- $\gamma$ & $0.23 \pm 0.06 \%$ & $0.08 \pm 0.03 \%$ \\
TGF- $\beta$ & $0.14 \pm 0.02 \%$ & $0.32 \pm 0.15 \%$ \\
IL-4 & $0.007 \pm 0.003 \%$ & $0.42 \pm 0.24 \%$ \\
IL-10 & $0.81 \pm 0.19 \%$ & $3.36 \pm 1.62 \%$ \\
\hline Cr & &
\end{tabular}

${ }^{51} \mathrm{Cr}$ chromium-51, NCRs natural cytotoxic receptors

${ }^{*} P<0.05 ;{ }^{* *} P<0.01 ;{ }^{* * *} P<0.001$ healthy donors $(n=8)$ vs. bladder cancer patients $(n=10)$

cytotoxicity. Moreover, the expansion of $\mathrm{CD} 56^{\text {bright }} \mathrm{CD} 16^{-}$ NK cells is likely to contribute to the enhanced NK cell-mediated cytotoxicity. Although the lytic activity of NK cells is generally attributed to the CD $56^{\text {dim }}$ subset, it is conceivable that the CD56 $6^{\text {bright }}$ subset becomes more mature and equally cytotoxic as the CD56 ${ }^{\mathrm{dim}}$ subset following cytokine stimulation. In line with this, Romee et al. [21] showed that IL-15 primed CD56 ${ }^{\text {bright }}$ NK cells with a highly potent antitumor activity in acute myeloid leukemia.

The phenotypic analysis of $\mathrm{BC}$ cells confirmed the high expression levels of several ligands recognized by NK activating receptors in both tumor cell subsets, making them suitable targets for NK cell-based immunotherapy. The levels of HLA class I molecules were not enough to protect tumor cells from NK-mediated lysis, suggesting that the susceptibility of $\mathrm{BC}$ cells might not entirely depend on missing self-recognition and that NK cell activation induced by activating ligands is a strong mechanism to overcome MHC class I inhibitory signals. This is consistent with a previous report showing that the loss of MHC inhibitory signals did not change the pattern of NK cell degranulation towards BC cells [22].

The decreased lytic activity observed in receptor blocking experiments confirmed the importance and cooperation pattern of DNAM-1- and NKG2D-dependent mechanisms in trigger activation signals and in overcoming the inhibitory signals resulting from MHC-I recognition, in both cell subsets. We cannot exclude that this alloreactivity of NK cells against tumor cells might also be caused by a killer-cell immunoglobulin-like receptor/HLA receptorligand mismatch, an aspect that was not explored in this work and deserves investigation.

Importantly, NK cells release critical factors that regulate the switch of spheres into a more differentiated status, thus reversing their resistance to cisplatin and indicating a dual effect on depletion of the CSC pool by direct killing and by generation of differentiated cells vulnerable to conventional therapies. This inducing-differentiation effect, already described and referred to as split energy, has been attributed to anergized NK cells that lose cytotoxicity but augmented the secretion of cytokine (IFN- $\gamma$, TNF- $\alpha$ ) inducers of CSC differentiation [19, 23].

NK cells from $\mathrm{BC}$ patients are less responsive to cytokine activation and display a reduced lytic activity, especially against CSCs, due to the low expression of NCRs and CD62L, crucial for recognition and killing of target cells, and by the presence of immature CD $57^{-} \mathrm{NK}$ cells. Apart from the modified NK phenotype, the increased levels of immunosuppressive cytokines (TGF- $\beta$, IL-10, and IL-4) and reduced expression of IFN- $\gamma /$ TNF- $\alpha$ prevented an effective antitumor immune response and abolished their differentiation-inducing effects on CSCs $[24,25]$. Finally, factors released by NK cells from BC patients were ineffective in driving differentiation of CSCs, which may contribute to the expansion of the CSC pool and subsequent tumor progression.

Recent studies suggest that malignant cells can bypass NK surveillance by releasing soluble forms of the NKG2D ligands, suppressing NK cell-mediated cytotoxicity. Marked levels of the soluble form of the MHC class I-related chain A, identified as a human NKG2D ligand, were found in the sera of patients with disseminated head-and-neck squamous cell [26] and human hepatocellular carcinomas [27] and neuroblastoma [28]. This tumor-derived soluble inhibitory ligand appears to be responsible for the downregulation of NKG2D expression in NK cells and subsequent impaired NKG2Dmediated cytotoxicity in patients with advanced disease. This mechanism also impairs the adaptive immunity due to the loss of allostimulatory capacity of dendritic cells mediated by NK cells [27]. Moreover, the downregulation of NKG2D was also reported in infiltrating and matched peripheral blood $\mathrm{T}$ cells in cancer patients with circulating tumor-derived soluble MHC class I-related chain A, suggesting this ligand can induce an impairment of the responsiveness of tumor antigen-specific effector $\mathrm{T}$ cells, leading to tumor escape from immunosurveillance [29]. The existence and nature of this immunosuppressive mechanism should be further explored in BC patients. 

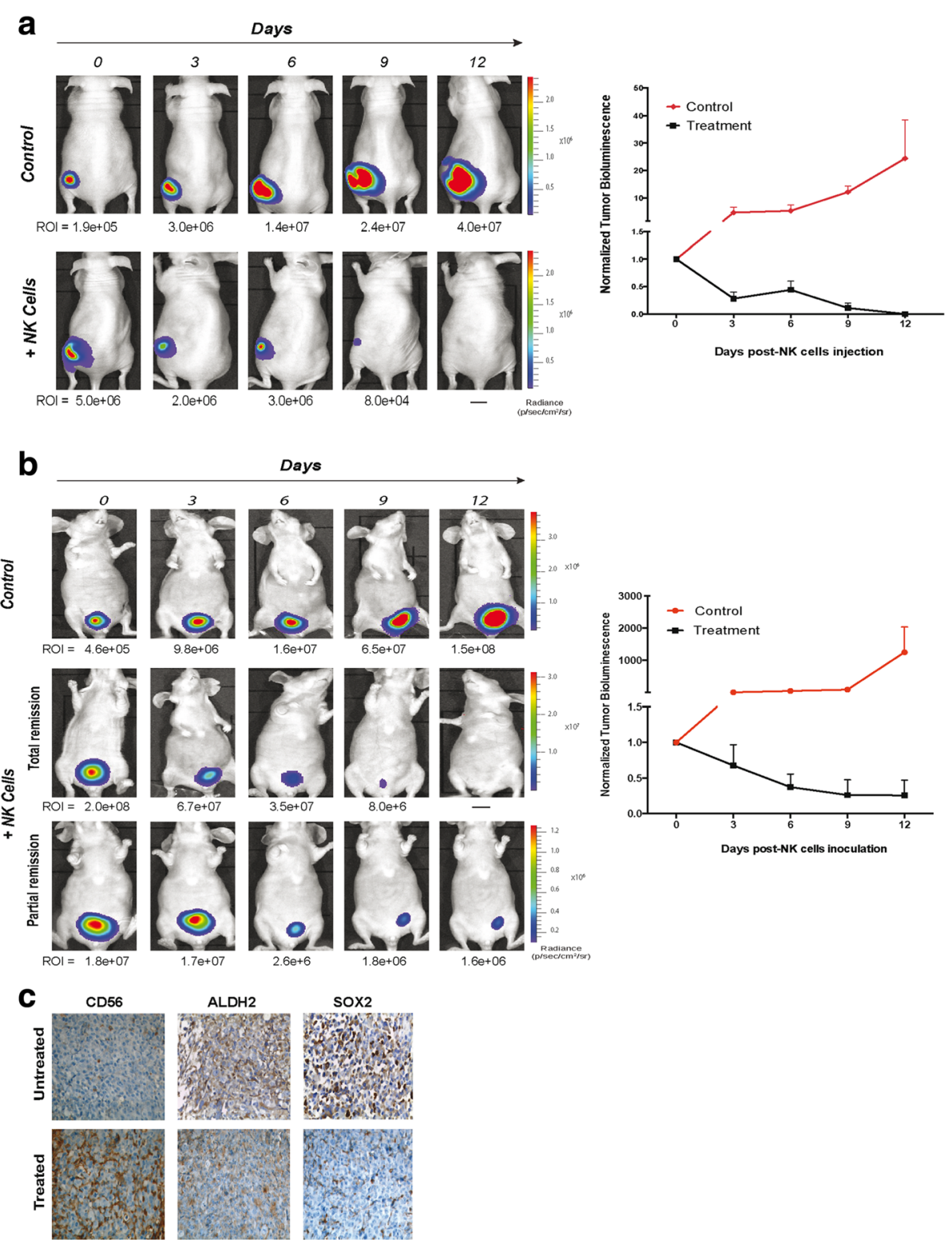

Fig. 5 Allogeneic activated-NK cells from healthy donors are effective in eradicating cancer stem-like cells in bladder cancer mouse models. a Serial bioluminescent images of a representative subcutaneous tumor-bearing mouse after intratumoral injection of activated-NK cells. b Anti-tumoral effects of activated-NK cells administered intravesically in mice bearing an orthotopic bladder cancer. All animals were treated twice a week with $5 \times 10^{6}$ NK cells for 2 weeks and were monitored each 3 days after treatment. The tumor size was evaluated by quantification of the bioluminescent signal (photons $/ \mathrm{sec} / \mathrm{cm}^{2} / \mathrm{sr}$ ) in a region of interest drawn around the tumor. The graphs represent tumor progression of untreated and treated animals normalized to the bioluminescent signal at the beginning of the treatment ( $n=5$, per group). $\mathbf{c}$ Immunohistochemical staining for $\mathrm{CD} 56, \mathrm{ALDH} 2$ and SOX2 in serial sections of an orthotopic untreated tumor (upper row) and in a residual treated tumor after the fourth intravesical administration of activated-NK cells (lower row). Original magnification: $\times 400$

These findings highlight the role of the tumor microenvironment in host immune response impairment and NK lytic function, and may explain the poor efficacy of adoptive transfer of autologous NK cells frequently observed in cancer patients with melanoma, lymphoma, and breast cancer due to NK-cell dysfunction [30-32]. Immunohistochemical analysis of $\mathrm{CD} 56^{+}$infiltrating-NK cells in biopsy specimens of $\mathrm{BC}$ patients revealed a poor tumor infiltration, independently of tumor stage and grade, reflecting an inefficient homing of NK cells in BC, unlikely to control tumor progression. Similar results were reported by Kripna et al. [33] in BC samples, suggesting tumor-infiltrating $\mathrm{NK}$ cells are not a prognostic factor in $\mathrm{BC}$, contrarily to other tumor types.

The in vivo studies showed a remarkable anti-tumor activity of healthy activated-NK cells in BC xenografted 
models. The intratumoral delivery of activated-NK cells leads to a complete abolishment of subcutaneous tumors with no evidence of recurrence, likely reflecting the ability of NK cells to kill CSCs and non-CSCs. In the orthotopic model, the intravesical administration of NK cells resulted in a massive decrease in the tumor burden, clearly demonstrating the intrinsic killing ability of NK cells in the tumor microenvironment. The considerable decreased expression of stemness markers in residual tumors confirm the CSC-targeting ability of NK cells, in addition to the elimination of differentiated tumor cells. We argue that this tremendous antitumor efficacy is largely related to the extensive tumor infiltration of NK cells, achieved via direct intravesical administration, surpassing the poor infiltration when delivered intravenously (data not shown). This has been observed in animal models and clinical studies, and is considered a critical factor for efficacious adoptive NK cell therapy when delivered systemically [34-36]. The innate ability of NK cells to target both stem and non-stem cell population by NK cells is of utmost importance to achieve a meaningful disease remission and survival benefits since non-stem cells might switch to a stem-like phenotype able to sustain tumor growth.

A limiting factor of this study is the use of immunocompromised mice lacking $\mathrm{T}$ cells that play a central role in tumor surveillance [37, 38]. It is well-known that NK cells, through release of cytokines, exchange bidirectional activating signals in a positive feedback with dendritic and $\mathrm{CD}^{+}{ }^{+} \mathrm{T}$ cells, with consequent enhancement of an antitumor immune response $[37,38]$. The lack of this synergistic effect with other immune effectors, only possible in an immunocompetent host, is likely to underestimate the anti-tumor response we have observed in our model, which was nevertheless notably significant. However, future studies using humanized mouse models to reproduce the complex interactions of NK cells with other cells of the immune system are needed to more accurately predict the anti-tumor clinical efficacy of allogeneic NK cell-based immunotherapy.

\section{Conclusions}

We demonstrated, for the first time, that intravesical therapy with ex vivo-activated allogeneic NK cells provides a rapid and noteworthy anti-tumoral response against BC by targeting both stem and non-stem cell populations. Importantly, the ability of NK cells to drive CSC differentiation, viewed as major precursors of muscle-invasive forms, are likely to prevent or delay recurrence and/or disease progression. These findings, although preclinical, provide evidence for the high therapeutic potential of NK cell-based adoptive immunotherapy in the eradication of bladder CSCs, an approach that should be exploited as part of a combinatorial therapeutic strategy in BC.

\section{Additional files}

Additional file 1: Table S1. Primer sequences used in real-time RT-qPCR analysis. (DOCX $16 \mathrm{~kb}$ )

Additional file 2: Figure S1. $\mid \mathrm{L}-2 / \mathrm{IL}-15$ increases the expression of NK cell-activating receptors. Representative FACS histograms of NK-activating receptors in resting and activated NK cells (gray and white profiles, respectively). Dotted lines represent isotype-matched controls. Bar graphs represent the mean \pm SEM $(n=3)$ of each receptor in fresh and activated NK cells. ${ }^{*} P<0.05$ and ${ }^{* *} P<0.01$ compared to freshly purified NK cells. (TIF $193 \mathrm{~kb}$ )

\section{Abbreviations}

ALDH: aldehyde dehydrogenase; BC: bladder cancer; CSCs: cancer stem cells; HD: healthy donors; MIBC: muscle-invasive bladder cancer; NCRs: natural cytotoxicity receptors; NK: natural killer; NMIBC: non-muscle-invasive bladder cancer

\section{Acknowledgments}

This work was supported by the Astellas European Foundation Uro-Oncology Grant 2013; Center of Investigation in Environment, Genetics and Oncobiology (CIMAGO) from the Faculty of Medicine of Coimbra (Refa 14/12), Portuguese Foundation for Science and Technology: Strategic Projects Pest-C/SAU/UI3282/ 2013, PEst-C/SAU/LA0001/2013-2014 and UID/NEU/04539/2013, and a Ph.D. scholarship attributed to Margarida Ferreira-Teixeira - SFRH/BD/77314/2011. We thank José Mário Ruivo, Paulo Teixeira, and Paula Neto for technical support in immunohistochemistry staining.

\section{Authors' contributions}

Study conception and design: CG, FR, PRS, and BP. Acquisition analysis and interpretation of data: MFT, DPO, BP, VA, VS, OC, CM, FR, PRS, and CG. Drafting and writing of the manuscript: MFT and CG. Critical revision of the manuscript for important intellectual content: CG, FR, PRS, BP, and CM. Study supervision: CG, FR, and PRS. All authors read and approved the final version of the manuscript.

\section{Competing interests}

The authors declare that they have no competing interests.

\section{Author details}

${ }^{1}$ Laboratory of Pharmacology and Experimental Therapeutics, Institute for Biomedical Imaging and Life Sciences (IBILI), Faculty of Medicine, University of Coimbra, Coimbra, Portugal. ${ }^{2}$ CNC.IBILI, University of Coimbra, Coimbra, Portugal. ${ }^{3}$ Urology and Renal Transplantation Department, Coimbra University Hospital Centre (CHUC), Coimbra, Portugal. ${ }^{4}$ Institute of Immunology, Faculty of Medicine, University of Coimbra, Coimbra, Portugal. ${ }^{5}$ Service of Anatomical Pathology, Coimbra University Hospital Centre (CHUC), Coimbra, Portugal. ${ }^{6}$ Institute of Anatomical and Molecular Pathology, Faculty of Medicine, University of Coimbra, Coimbra, Portugal. ${ }^{7}$ Viral Immunobiology, Institute of Experimental Immunology, University of Zürich, Zürich, Switzerland. ${ }^{8}$ Center of Investigation in Environment, Genetics and Oncobiology (CIMAGO), Faculty of Medicine, University of Coimbra, Coimbra, Portugal. ${ }^{9}$ Immunology and Oncology Laboratory, Center for Neurosciences and Cell Biology (CNC), University of Coimbra, Coimbra, Portugal.

Received: 31 May 2016 Accepted: 6 October 2016

Published online: 21 October 2016

\section{References}

1. Anastasiadis A, de Reijke TM. Best practice in the treatment of nonmuscle invasive bladder cancer. Ther Adv Urol. 2012;4:13-32.

2. Knowles MA, Hurst CD. Molecular biology of bladder cancer: new insights into pathogenesis and clinical diversity. Nat Rev Cancer. 2015;15:25-41.

3. Brandt WD, Matsui W, Rosenberg JE, He X, Ling S, Schaeffer EM, et al. Urothelial carcinoma: stem cells on the edge. Cancer Metastasis Rev. 2009;28:291-304.

4. Volkmer JP, Sahoo D, Chin RK, Ho PL, Tang C, Kurtova AV, et al. Three differentiation states risk-stratify bladder cancer into distinct subtypes. Proc Natl Acad Sci U S A. 2012;109:2078-83. 
5. Ferreira-Teixeira M, Parada B, Rodrigues-Santos P, Alves V, Ramalho JS, Caramelo $F$, et al. Functional and molecular characterization of cancer stem-like cells in bladder cancer: a potential signature for muscle-invasive tumors. Oncotarget. 2015;6:36185-201.

6. Zhang Y, Wang Z, Yu J, Shi J, Wang C, Fu W, et al. Cancer stem-like cells contribute to cisplatin resistance and progression in bladder cancer. Cancer Lett. 2012:322:70-7.

7. Chan KS, Espinosa I, Chao M, Wong D, Ailles L, Diehn M, et al. Identification, molecular characterization, clinical prognosis, and therapeutic targeting of human bladder tumor-initiating cells. Proc Natl Acad Sci U S A. 2009;106: 14016-21.

8. Robertson MJ, Ritz J. Biology and clinical relevance of human natural killer cells. Blood. 1990;76:2421-38.

9. Salagianni M, Baxevanis CN, Papamichail M, Perez SA. New insights into the role of NK cells in cancer immunotherapy. Oncoimmunology. 2012;1:205-7.

10. Wang R, Jaw JJ, Stutzman NC, Zou Z, Sun PD. Natural killer cell-produced IFN-gamma and TNF-alpha induce target cell cytolysis through up-regulation of ICAM-1. J Leukoc Biol. 2012;91:299-309.

11. Vivier E, Raulet $\mathrm{DH}$, Moretta A, Caligiuri MA, Zitvogel L, Lanier $L L$, et al. Innate or adaptive immunity? The example of natural killer cells. Science. 2011;331:44-9.

12. Yoon SR, Kim TD, Choi I. Understanding of molecular mechanisms in natural killer cell therapy. Exp Mol Med. 2015;47, e141.

13. Lanier LL. Activating and inhibitory NK cell receptors. Adv Exp Med Biol. 1998:452:13-8.

14. Bae DS, Hwang YK, Lee JK. Importance of NKG2D-NKG2D ligands interaction for cytolytic activity of natural killer cell. Cell Immunol. 2012;276:122-7.

15. Pietra G, Manzini C, Vitale M, Balsamo M, Ognio E, Boitano M, et al. Natural killer cells kill human melanoma cells with characteristics of cancer stem cells. Int Immunol. 2009;21:793-801.

16. Wu A, Wiesner S, Xiao J, Ericson K, Chen W, Hall WA, et al. Expression of MHCI and NK ligands on human CD133+ glioma cells: possible targets of immunotherapy. J Neurooncol. 2007:83:121-31.

17. Maccalli C, Volonte A, Cimminiello C, Parmiani G. Immunology of cancer stem cells in solid tumours. A review. Eur J Cancer. 2014;50:649-55.

18. Hasmim M, Messai Y, Ziani L, Thiery J, Bouhris JH, Noman MZ, et al. Critical role of tumor microenvironment in shaping NK cell functions: implication of hypoxic stress. Front Immunol. 2015;6:482

19. Jewett A, Man YG, Tseng HC. Dual functions of natural killer cells in selection and differentiation of stem cells; role in regulation of inflammation and regeneration of tissues. J Cancer. 2013;4:12-24.

20. Eble JN, Sauter G, Epstein J, Sesterhenn IA (Eds.): World Health Organization Classification of Tumours. Pathology and Genetics of Tumours of the Urinary System and Male Genital Organs. IARC Press: Lyon 2004.

21. Romee R, Leong JW, Schneider SE, Sullivan RP, Fehniger TA. IL-15 primes a highly potent anti-leukemia response by CD56bright NK cells. Blood. 2013; 122:2283.

22. Garcia-Cuesta EM, Lopez-Cobo S, Alvarez-Maestro M, Esteso G, Romera-Cardenas G, Rey $M$, et al. NKG2D is a key receptor for recognition of bladder cancer cells by IL-2-activated NK cells and BCG promotes NK cell activation. Front Immunol. 2015;6:284.

23. Tseng HC, Cacalano N, Jewett A. Split anergized Natural Killer cells halt inflammation by inducing stem cell differentiation, resistance to NK cell cytotoxicity and prevention of cytokine and chemokine secretion. Oncotarget. 2015:6:8947-59.

24. Kaiko GE, Horvat JC, Beagley KW, Hansbro PM. Immunological decision-making: how does the immune system decide to mount a helper T-cell response? Immunology. 2008;123:326-38.

25. Joyce S. Natural T cells: cranking up the immune system by prompt cytokine secretion. Proc Natl Acad Sci U S A. 2000;97:6933-5.

26. Klöß S, Chambron N, Gardlowski T, Arseniev L, Koch J, Esser R, et al. Increased sMICA and TGF $\beta(1)$ levels in HNSCC patients impair NKG2D-dependent functionality of activated NK cells. Oncoimmunology. 2015;4, e1055993.

27. Jinushi M, Takehara T, Tatsumi T, Hiramatsu N, Sakamori R, Yamaguchi S, et al. Impairment of natural killer cell and dendritic cell functions by the soluble form of $\mathrm{MHC}$ class I-related chain A in advanced human hepatocelular carcinomas. J Hepatol. 2005;43:1013-20.

28. Kloess S, Huenecke S, Piechulek D, Esser R, Koch J, Brehm C, et al. IL-2-activated haploidentical NK cells restore NKG2D-mediated NK-cell cytotoxicity in neuroblastoma patients by scavenging of plasma MICA. Eur J Immunol. 2010;40:3255-67.
29. Groh V, Wu J, Yee C, Spies T. Tumour-derived soluble MIC ligands impair expression of NKG2D and T-cell activation. Nature. 2002:419:734-8.

30. Cheng M, Chen Y, Xiao W, Sun R, Tian Z. NK cell-based immunotherapy for malignant diseases. Cell Mol Immunol. 2013;10:230-52.

31. Parkhurst MR, Riley JP, Dudley ME, Rosenberg SA. Adoptive transfer of autologous natural killer cells leads to high levels of circulating natural killer cells but does not mediate tumor regression. Clin Cancer Res. 2011;17:6287-97.

32. Krause SW, Gastpar R, Andreesen R, Gross C, Ullrich H, Thonigs G, et al. Treatment of colon and lung cancer patients with ex vivo heat shock protein 70-peptide-activated, autologous natural killer cells: a clinical phase I trial. Clin Cancer Res. 2004:10:3699-707.

33. Krpina K, Babarovic E, Spanjol J, Dordevic G, Maurer T, Jonjic N. Correlation of tumor-associated macrophages and NK cells with bladder cancer size and T stage in patients with solitary low-grade urothelial carcinoma. Wien Klin Wochenschr. 2016;128:248-52.

34. Du X, Jin R, Ning N, Li L, Wang Q, Liang W, et al. In vivo distribution and antitumor effect of infused immune cells in a gastric cancer model. Oncol Rep. 2012;28:1743-9.

35. Levy EM, Roberti MP, Mordoh J. Natural killer cells in human cancer: from biological functions to clinical applications. J Biomed Biotechnol. 2011;2011:676198.

36. Esendagli G, Bruderek K, Goldmann T, Busche A, Branscheid D, Vollmer E, et al. Malignant and non-malignant lung tissue areas are differentially populated by natural killer cells and regulatory T cells in non-small cell lung cancer. Lung Cancer. 2008;59:32-40.

37. Ferlazzo G, Munz C. NK cell compartments and their activation by dendritic cells. J Immunol. 2004;172:1333-9.

38. Gerosa F, Baldani-Guerra B, Nisii C, Marchesini V, Carra G, Trinchieri G. Reciprocal activating interaction between natural killer cells and dendritic cells. J Exp Med. 2002;195:327-33.

\section{Submit your next manuscript to BioMed Central and we will help you at every step:}

- We accept pre-submission inquiries

- Our selector tool helps you to find the most relevant journal

- We provide round the clock customer support

- Convenient online submission

- Thorough peer review

- Inclusion in PubMed and all major indexing services

- Maximum visibility for your research

Submit your manuscript at www.biomedcentral.com/submit 